\title{
Some Identities Involving the Reciprocal Sums of One-Kind Chebyshev Polynomials
}

\author{
Yuankui $\mathrm{Ma}^{1}$ and Xingxing $\mathrm{Lv}^{2}$ \\ ${ }^{1}$ School of Science, Xian Technological University, Xi'an, Shaanxi, China \\ ${ }^{2}$ School of Mathematics, Northwest University, Xian, Shaanxi, China \\ Correspondence should be addressed to Xingxing Lv; lvxingxing@stumail.nwu.edu.cn
}

Received 10 May 2017; Accepted 11 June 2017; Published 11 July 2017

Academic Editor: Francesco Marotti de Sciarra

Copyright (c) 2017 Yuankui Ma and Xingxing Lv. This is an open access article distributed under the Creative Commons Attribution License, which permits unrestricted use, distribution, and reproduction in any medium, provided the original work is properly cited.

We use the elementary and analytic methods and the properties of Chebyshev polynomials to study the computational problem of the reciprocal sums of one-kind Chebyshev polynomials and give several interesting identities for them. At the same time, we also give a general computational method for this kind of reciprocal sums.

\section{Introduction}

It is well known that Chebyshev polynomials of the first and second kind $T_{n}(x)$ and $U_{n}(x)$ are defined as follows: $T_{0}(x)=$ $1, T_{1}(x)=x$, and the recursion formula $T_{n+1}(x)=2 x T_{n}(x)-$ $T_{n-1}(x)$ for all integers $n \geq 1 . U_{0}(x)=1, U_{1}(x)=2 x$, and the recursion formula $U_{n+1}(x)=2 x U_{n}(x)-U_{n-1}(x)$ for all integers $n \geq 1$. The generation functions of these polynomials are

$$
\begin{aligned}
& \frac{1-x t}{1-2 x t+t^{2}}=\sum_{n=1}^{\infty} T_{n}(x) t^{n}, \quad(|x|<1,|t|<1), \\
& \frac{1}{1-2 x t+t^{2}}=\sum_{n=1}^{\infty} U_{n}(x) t^{n}, \quad(|x|<1,|t|<1) .
\end{aligned}
$$

The general term formulae of $T_{n}(x)$ and $U_{n}(x)$ are expressed as

$$
\begin{aligned}
& T_{n}(x)=\frac{1}{2}\left[\left(x+\sqrt{x^{2}-1}\right)^{n}+\left(x-\sqrt{x^{2}-1}\right)^{n}\right] \\
& U_{n}(x)=\frac{1}{2 \sqrt{x^{2}-1}}\left[\left(x+\sqrt{x^{2}-1}\right)^{n+1}\right. \\
& \left.\quad-\left(x-\sqrt{x^{2}-1}\right)^{n+1}\right] .
\end{aligned}
$$

If we take $x=\cos \theta$, then

$$
\begin{aligned}
T_{n}(\cos \theta) & =\cos (n \theta), \\
U_{n}(\cos \theta) & =\frac{\sin ((n+1) \theta)}{\sin \theta} .
\end{aligned}
$$

Since all these definitions and properties of Chebyshev polynomials can be found in any handbook of mathematics, there is no need to list the source everywhere.

Recently, some authors studied the properties of Chebyshev polynomials and obtained many interesting conclusions. For example, Li [1] obtained some identities involving power sums of $T_{n}(x)$ and $U_{n}(x)$. As some applications of these results, she obtained some divisibility properties involving Chebyshev polynomials. At the same time, she also proposed the following open problem.

Whether there exists an exact expression for the derivative or integral of the Chebyshev polynomials of the first kind in terms of the Chebyshev polynomials of the first kind (and vice-versa) is the question.

Wang and Zhang [2] and Zhang and Wang [3] partly solved these problems. Some theoretical results related to Chebyshev polynomials can be found in Ma and Zhang [4], Cesarano [5], Babusci et al. [6-8], Lee and Wong [9], and Wang and Han [10]. Doha and others [11-14] and Bircan and Pommerenke [15] also obtained many important applications of the Chebyshev polynomials. 
In this paper, we consider the computational problem of the reciprocal sums of Chebyshev polynomials. That is, let $q$ and $k$ be positive integers with $q \geq 3$, for any real number $x$; if $T_{n}(x) \neq 0$, then we consider the computational problem of the summations

$$
\begin{aligned}
& \sum_{a=1}^{q-1} \frac{1}{T_{a}^{2 k}(x)} \\
& \sum_{a=1}^{q-1} \frac{1}{U_{a-1}^{2 k}(x)}
\end{aligned}
$$

Although there are many results related to Chebyshev polynomials, it seems that none had studied the computational problem of (4). The main reason may be that a computational formula does not exist. But for some special real number $x$, we can really get the precise value of (4). In this paper, we will illustrate this point. That is, we will use the elementary and analytic methods and the properties of Chebyshev polynomials to prove the following results.

Theorem 1. Let $q$ be an integer with $q \geq 3$. Then for any integer $h$ with $(h, q)=1$, one has the identities

$$
\begin{aligned}
& \sum_{a=1}^{q-1} \frac{1}{U_{a-1}^{2}(\cos (\pi h / q))}=\frac{\sin ^{2}(\pi h / q)}{3}\left(q^{2}-1\right) \\
& \sum_{a=1}^{q-1} \frac{1}{U_{a-1}^{4}(\cos (\pi h / q))} \\
& \quad=\frac{\sin ^{4}(\pi h / q)}{45}\left(q^{2}-1\right)\left(q^{2}+11\right) ; \\
& \sum_{a=1}^{q-1} \frac{1}{U_{a-1}^{6}(\cos (\pi h / q))} \\
& \quad=\frac{\sin ^{6}(\pi h / q)}{945}\left(q^{2}-1\right)\left(2 q^{2}-11\right)\left(q^{2}+17\right) .
\end{aligned}
$$

Theorem 2. Let $q$ be an odd number with $q \geq 3$. Then for any integer $h$ with $(h, q)=1$, one has the identities

$$
\begin{aligned}
& \sum_{a=1}^{q-1} \frac{1}{T_{a}^{2}(\cos (\pi h / q))}=q^{2}-1 \\
& \sum_{a=1}^{q-1} \frac{1}{T_{a}^{4}(\cos (\pi h / q))}=\frac{1}{3}\left(q^{2}-1\right)\left(q^{2}+3\right) \\
& \sum_{a=1}^{q-1} \frac{1}{T_{a}^{6}(\cos (\pi h / q))}=\frac{1}{15}\left(q^{2}-1\right)\left(2 q^{4}+7 q^{2}-363\right)
\end{aligned}
$$

Some Notes. First in Theorem 2, we must limit $q$ as an odd number. Otherwise, if $q$ is an even number, then $a=(1 / 2) \cdot q$ is an integer, $1 \leq a \leq q-1$ and $\cos (\pi a / q)=\cos (\pi / 2)=0$. Therefore, the fraction $1 / T_{a}^{2 k}(\cos (\pi a / q))$ is meaningless.

Second, for any positive integer $k$ and $x=\cos (\pi h / q)$ with $(h, q)=1$, we can give an computational formula for (4). Of course, the calculation is very complicated when $k$ is larger. So we do not give a general conclusion for (4), only give an efficient calculating method. In fact if we use computer MatLab program, and by means of recursive method in Lemma 4, we can also obtain all precise values of (4) for any positive integer $k$.

\section{Several Lemmas}

To complete the proofs of our theorems, we need following lemmas. First we have

Lemma 3. Let $q>3$ be an integer. Then for variable $s$ with $0<s<1$ and function $f(\pi s)=\ln \sin (\pi s)$, one has the identity

$$
\sum_{a=1}^{q-1} f^{(2 k)}\left(\frac{\pi a}{q}\right)=\frac{(-1)^{k} \cdot 2^{2 k-1} \cdot B_{2 k}}{k} \cdot\left(q^{2 k}-1\right)
$$

where $f^{(n)}(s)$ denotes the $n$-order derivative of $f(s), B_{2 k}$ is Bernoulli numbers.

Proof. For any real number $s$, from Corollary 6 (Section 3, Chapter 5) in [16] we have the identity

$$
\sin (\pi s)=\pi s \prod_{n=1}^{\infty}\left(1-\frac{s^{2}}{n^{2}}\right) .
$$

Then from (8), the definition and properties of derivative we have

$$
\begin{aligned}
& \pi f^{\prime}(\pi s)=\frac{1}{s}+\sum_{n=1}^{\infty}\left(\frac{1}{n+s}-\frac{1}{n-s}\right) \\
& \pi f^{\prime \prime}(\pi s)=-\frac{1}{s^{2}}-\sum_{n=1}^{\infty}\left(\frac{1}{(n+s)^{2}}+\frac{1}{(n-s)^{2}}\right) .
\end{aligned}
$$

Generally, for any positive integer $k$, we have

$$
\begin{aligned}
\pi^{2 k} f^{(2 k)}(\pi s)= & -\frac{(2 k-1) !}{s^{2 k}} \\
& -\sum_{n=1}^{\infty}\left(\frac{(2 k-1) !}{(n+s)^{2 k}}+\frac{(2 k-1) !}{(n-s)^{2 k}}\right) .
\end{aligned}
$$


Now taking $s=a / q$ in (10), and summation for all $1 \leq a \leq$ $q-1$ and noting the definition and properties of complete residue system $\bmod q$ (see [16]) we have

$$
\begin{aligned}
\pi^{2 k} \sum_{a=1}^{q-1} f^{(2 k)}\left(\frac{\pi a}{q}\right) & \\
= & -(2 k-1) ! \sum_{a=1}^{q-1} \frac{q^{2 k}}{a^{2 k}} \\
& -\sum_{a=1}^{q-1} \sum_{n=1}^{\infty}\left(\frac{(2 k-1) !}{(n+a / q)^{2 k}}+\frac{(2 k-1) !}{(n-a / q)^{2 k}}\right) \\
= & -q^{2 k} \sum_{a=1}^{q-1} \sum_{n=0}^{\infty} \frac{(2 k-1) !}{(q n+a)^{2 k}}-(2 k-1) ! \sum_{a=1}^{q-1} \frac{q^{2 k}}{(q-a)^{2 k}} \\
& -q^{2 k} \sum_{a=1}^{q-1} \sum_{n=2}^{\infty} \frac{(2 k-1) !}{(q n-a)^{2 k}} \\
= & -2 q^{2 k} \sum_{a=1}^{q-1} \sum_{n=0}^{\infty} \frac{(2 k-1) !}{(q n+a)^{2 k}} \\
= & -2(2 k-1) !\left(\sum_{n=1}^{\infty} \frac{q^{2 k}}{n^{2 k}}-\sum_{n=1}^{\infty} \frac{1}{n^{2 k}}\right) \\
= & \left(q^{2 k}-1\right) \zeta(2 k) . \\
= & -2 k-1) \\
= &
\end{aligned}
$$

Note that Riemann $\zeta$-function $\zeta(2 k)=\sum_{n=1}^{\infty}\left(1 / n^{2 k}\right)=$ $(-1)^{k+1}\left((2 \pi)^{2 k} B_{2 k} / 2(2 k)\right.$ !) (see [17], Theorem 12.17). Then from (11) we have

$$
\begin{aligned}
\pi^{2 k} \sum_{a=1}^{q-1} f^{(2 k)}\left(\frac{\pi a}{q}\right)= & -2(2 k-1) !\left(q^{2 k}-1\right) \\
& \cdot(-1)^{k+1} \frac{(2 \pi)^{2 k} B_{2 k}}{2(2 k) !} \\
= & \frac{(-1)^{k} \cdot(2 \pi)^{2 k} \cdot B_{2 k}}{2 k} \cdot\left(q^{2 k}-1\right) .
\end{aligned}
$$

This proves Lemma 3.

Lemma 4. Let $f(s)=\ln \sin (\pi s), \alpha=\alpha(s)=\cot (\pi s)$. Then one has

$$
\begin{aligned}
& f^{\prime \prime}(s)=-\pi^{2}\left(1+\alpha^{2}\right) \\
& f^{(4)}(s)=-\pi^{4}\left(6 \alpha^{4}+8 \alpha^{2}+2\right) \\
& f^{(6)}(s)=-\pi^{6}\left(120 \alpha^{6}+240 \alpha^{4}+136 \alpha^{2}+16\right) .
\end{aligned}
$$

Proof. Noting that the identity $1+\cot ^{2} s=1 / \sin ^{2} s$, from the definition and properties of derivative we have $f^{\prime}(s)=$ $\pi \cot (\pi s)=\pi \alpha$ and

$$
\begin{aligned}
f^{\prime \prime}(s) & =-\frac{\pi^{2}}{\sin (\pi s)}=-\pi^{2}\left(1+\cot ^{2}(\pi s)\right) \\
& =-\pi^{2}\left(1+\alpha^{2}\right) .
\end{aligned}
$$

This proves the first formula of Lemma 4.

Similarly, we have

$$
\begin{aligned}
& f^{(3)}(s)=-2 \pi^{2} \alpha \alpha^{\prime}=2 \pi^{3} \alpha+2 \pi^{3} \alpha^{3}, \\
& f^{(4)}(s)=2 \pi^{3} \alpha^{\prime}+6 \pi^{3} \alpha^{2} \alpha^{\prime}=-\pi^{4}\left(6 \alpha^{4}+8 \alpha^{2}+2\right) .
\end{aligned}
$$

This is the second formula of Lemma 4.

It is easy to prove that

$$
f^{(6)}(s)=-\pi^{6}\left(120 \alpha^{6}+240 \alpha^{4}+136 \alpha^{2}+16\right) .
$$

This completes the proof of Lemma 4 .

Lemma 5. Let $q$ be an integer with $q \geq 3$. Then for any integer $h$ with $(h, q)=1$, one has the identities

$$
\begin{aligned}
& \sum_{a=1}^{q-1} \frac{1}{\sin ^{2}(\pi h a / q)}=\frac{1}{3}(q-1)(q+1) ; \\
& \sum_{a=1}^{q-1} \frac{1}{\sin ^{4}(\pi h a / q)}=\frac{1}{45}\left(q^{2}-1\right)\left(q^{2}+11\right) ; \\
& \sum_{a=1}^{q-1} \frac{1}{\sin ^{6}(\pi h a / q)} \\
& =\frac{1}{945}\left(q^{2}-1\right)\left(q^{2}+17\right)\left(2 q^{2}-11\right) .
\end{aligned}
$$

Proof. Since $(h, q)=1$, if $a$ pass through a complete residue system $\bmod q$, then ha also pass through a complete residue system $\bmod q$. Therefore, without loss of generality we can assume that $h=1$. Noting that $B_{2}=1 / 6$ and $1+\cot ^{2}(x)=$ $1 / \sin ^{2}(x)$, from Lemmas 3 and 4 with $k=1$ we have

$$
\begin{aligned}
& -\sum_{a=1}^{q-1}\left(1+\cot ^{2}\left(\frac{\pi a}{q}\right)\right)=-\sum_{a=1}^{q-1} \frac{1}{\sin ^{2}(\pi a / q)} \\
& =-\frac{1}{3}\left(q^{2}-1\right)
\end{aligned}
$$

or

$$
\sum_{a=1}^{q-1} \frac{1}{\sin ^{2}(\pi a / q)}=\frac{1}{3}(q-1)(q+1) .
$$

Similarly, noting that $B_{4}=-1 / 30$, from Lemmas 3 and 4 with $k=2$ and applying (19) we have

$$
\begin{aligned}
& -\sum_{a=1}^{q-1}\left(6 \cot ^{4}\left(\frac{\pi a}{q}\right)+8 \cot ^{2}\left(\frac{\pi a}{q}\right)+2\right) \\
& =-\frac{2}{15}\left(q^{4}-1\right),
\end{aligned}
$$


which implies that

$$
\begin{aligned}
& \sum_{a=1}^{q-1}\left[6\left(\frac{1}{\sin ^{2}(\pi a / q)}-1\right)^{2}+8\left(\frac{1}{\sin ^{2}(\pi a / q)}-1\right)\right. \\
& +2]=\frac{2}{15}\left(q^{4}-1\right)
\end{aligned}
$$

or

$$
\sum_{a=1}^{q-1} \frac{1}{\sin ^{4}(\pi a / q)}=\frac{1}{45}\left(q^{2}-1\right)\left(q^{2}+11\right) .
$$

Noting that $B_{6}=1 / 42$, from Lemmas 3 and 4 with $k=3$, applying (19) and (22) we have

$$
\begin{aligned}
& -\sum_{a=1}^{q-1}\left(120 \cot ^{6}\left(\frac{\pi a}{q}\right)+240 \cot ^{4}\left(\frac{\pi a}{q}\right)\right. \\
& \left.+136 \cot ^{2}\left(\frac{\pi a}{q}\right)+16\right)=-\frac{16}{63}\left(q^{6}-1\right)
\end{aligned}
$$

or

$$
\begin{aligned}
& \sum_{a=1}^{q-1}\left[15\left(\frac{1}{\sin ^{2}(\pi a / q)}-1\right)^{3}\right. \\
& +30\left(\frac{1}{\sin ^{2}(\pi a / q)}-1\right)^{2}+17\left(\frac{1}{\sin ^{2}(\pi a / q)}-1\right) \\
& +2]=\frac{2}{63}\left(q^{6}-1\right),
\end{aligned}
$$

which implies that

$$
\sum_{a=1}^{q-1} \frac{1}{\sin ^{6}(\pi a / q)}=\frac{1}{945}\left(q^{2}-1\right)\left(q^{2}+17\right)\left(2 q^{2}-11\right) .
$$

Now Lemma 5 follows from (19), (22), and (25).

In fact, by using Lemma 4 and the method of proving Lemma 5 we can give a computational formula for

$$
\sum_{a=1}^{q-1} \frac{1}{\sin ^{2 k}(\pi a / q)}
$$

with all positive integer $k$. Here just in order to meet the demands of main results we only calculated $k=1,2$, and 3.

\section{Proofs of the Theorems}

In this section, we shall complete the proofs of our theorems. First we prove Theorem 1. For any integer $q \geq 3$, taking $x=$ $\cos (\pi h / q)$ with $(h, q)=1$, from (3) we have

$$
\begin{aligned}
\sum_{a=1}^{q-1} \frac{1}{U_{a-1}^{2 k}(x)} & =\sum_{a=1}^{q-1} \frac{1}{U_{a-1}^{2 k}(\cos (\pi h / q))} \\
& =\sin ^{2 k}\left(\frac{\pi h}{q}\right) \sum_{a=1}^{q-1} \frac{1}{\sin ^{2 k}(\pi h a / q)} .
\end{aligned}
$$

Now Theorem 1 follows from (27) and Lemma 5 with $k=1,2$, and 3.

To prove Theorem 2, we note that, for any odd number $q \geq 3$, if $a$ pass through a complete residue system $\bmod q$, then $2 a$ also pass through a complete residue system $\bmod q$. So from the properties of trigonometric functions we have

$$
\begin{aligned}
\sum_{a=1}^{q-1} \frac{1}{\sin ^{2}(\pi a / q)}= & \sum_{a=1}^{q-1} \frac{1}{\sin ^{2}(2 \pi a / q)} \\
= & \sum_{a=1}^{q-1} \frac{\sin ^{2}(\pi a / q)+\cos ^{2}(\pi a / q)}{4 \sin ^{2}(\pi a / q) \cos ^{2}(\pi a / q)} \\
= & \frac{1}{4} \sum_{a=1}^{q-1} \frac{1}{\sin ^{2}(\pi a / q)} \\
& +\frac{1}{4} \sum_{a=1}^{q-1} \frac{1}{\cos ^{2}(\pi a / q)} .
\end{aligned}
$$

From (3), (28), and Lemma 5 we may immediately deduce that

$$
\begin{aligned}
\sum_{a=1}^{q-1} \frac{1}{T_{a}^{2}(\cos (\pi h / q))} & =\sum_{a=1}^{q-1} \frac{1}{\cos ^{2}(\pi h a / q)} \\
& =\sum_{a=1}^{q-1} \frac{3}{\sin ^{2}(\pi a / q)}=q^{2}-1 .
\end{aligned}
$$

Similarly, we also have

$$
\begin{aligned}
\sum_{a=1}^{q-1} \frac{1}{\sin ^{4}(\pi a / q)}= & \sum_{a=1}^{q-1} \frac{1}{\sin ^{4}(2 \pi a / q)} \\
= & \sum_{a=1}^{q-1} \frac{\left(\sin ^{2}(\pi a / q)+\cos ^{2}(\pi a / q)\right)^{2}}{16 \sin ^{4}(\pi a / q) \cos ^{4}(\pi a / q)} \\
= & \frac{1}{16} \sum_{a=1}^{q-1} \frac{1}{\sin ^{4}(\pi a / q)} \\
& +\frac{1}{16} \sum_{a=1}^{q-1} \frac{1}{\cos ^{4}(\pi a / q)} \\
& +\frac{1}{2} \sum_{a=1}^{q-1} \frac{1}{\sin ^{2}(2 \pi a / q)} .
\end{aligned}
$$

So from (30) and Lemma 5 we have

$$
\begin{aligned}
\sum_{a=1}^{q-1} \frac{1}{\cos ^{4}(\pi a / q)} & =\sum_{a=1}^{q-1} \frac{15}{\sin ^{4}(\pi a / q)}-\sum_{a=1}^{q-1} \frac{8}{\sin ^{2}(\pi a / q)} \\
& =\frac{1}{3}\left(q^{2}-1\right)\left(q^{2}+3\right) .
\end{aligned}
$$


Combining (3) and (31) we have the identity

$$
\begin{aligned}
\sum_{a=1}^{q-1} \frac{1}{T_{a}^{4}(\cos (\pi h / q))} & =\sum_{a=1}^{q-1} \frac{1}{\cos ^{4}(\pi h a / q)} \\
& =\frac{1}{3}\left(q^{2}-1\right)\left(q^{2}+3\right) .
\end{aligned}
$$

From the method of proving (30) we also have

$$
\begin{aligned}
\sum_{a=1}^{q-1} \frac{1}{\sin ^{6}(\pi a / q)}= & \sum_{a=1}^{q-1} \frac{1}{\sin ^{6}(2 \pi a / q)} \\
= & \sum_{a=1}^{q-1} \frac{\left(\sin ^{2}(\pi a / q)+\cos ^{2}(\pi a / q)\right)^{3}}{64 \sin ^{6}(\pi a / q) \cos ^{6}(\pi a / q)} \\
= & \frac{1}{64} \sum_{a=1}^{q-1} \frac{1}{\sin ^{6}(\pi a / q)} \\
& +\frac{1}{64} \sum_{a=1}^{q-1} \frac{1}{\cos ^{6}(\pi a / q)} \\
& +\frac{3}{4} \sum_{a=1}^{q-1} \frac{1}{\sin ^{4}(2 \pi a / q)} .
\end{aligned}
$$

From (3), Lemma 5, and (33) we can deduce that

$$
\begin{aligned}
\sum_{a=1}^{q-1} \frac{1}{T_{a}^{6}(\cos (\pi h / q))} & =\sum_{a=1}^{q-1} \frac{1}{\cos ^{6}(\pi h a / q)} \\
& =\sum_{a=1}^{q-1} \frac{63}{\sin ^{6}(\pi h a / q)} \\
& -\sum_{a=1}^{q-1} \frac{48}{\sin ^{4}(\pi h a / q)} \\
& =\frac{\left(q^{2}-1\right)\left(2 q^{4}+7 q^{2}-363\right)}{15} .
\end{aligned}
$$

Now Theorem 2 follows from (29), (32), and (34).

This completes all proofs of our results.

\section{Conflicts of Interest}

The authors declare that there are no conflicts of interest regarding the publication of this paper.

\section{Acknowledgments}

This work is supported by NSF (11371291) of China and the Scientific Research Program Funded by Shaanxi Provincial Education Department (no. 16JK1373).

\section{References}

[1] X. Li, "Some identities involving Chebyshev polynomials," Mathematical Problems in Engineering, vol. 2015, Article ID 950695, 5 pages, 2015.
[2] T. Wang and H. Zhang, "Some identities involving the derivative of the first kind Chebyshev polynomials," Mathematical Problems in Engineering, vol. 2015, Article ID 146313, 7 pages, 2015.

[3] W. Zhang and T. Wang, "Two identities involving the integral of the first kind Chebyshev polynomials," Bulletin Mathematique de la Societe des Sciences Mathematiques de Roumanie, vol. 60, pp. 91-98, 2017.

[4] R. Ma and W. Zhang, "Several identities involving the Fibonacci numbers and Lucas numbers," The Fibonacci Quarterly, vol. 45, no. 2, pp. 164-170, 2007.

[5] C. Cesarano, "Identities and generating functions on Chebyshev polynomials," Georgian Mathematical Journal, vol. 19, no. 3, pp. 427-440, 2012.

[6] D. Babusci, G. Dattoli, E. Di Palma, and E. Sabia, "Chebyshev polynomials and generalized complex numbers," Advances in Applied Clifford Algebras, vol. 24, no. 1, pp. 1-10, 2014.

[7] G. Dattoli, D. Sacchetti, and C. Cesarano, "A note on Chebyshev polynomials," Annali dell'Università di Ferrara. Nuova Serie. Sezione VII. Scienze Matematiche, vol. 47, pp. 107-115, 2001.

[8] G. Dattoli, "Integral transforms and Chebyshev-like polynomials," Applied Mathematics and Computation, vol. 148, no. 1, pp. 225-234, 2004.

[9] C.-L. Lee and K. B. Wong, “On Chebyshev's polynomials and certain combinatorial identities," Bulletin of the Malaysian Mathematical Sciences Society, vol. 34, no. 2, pp. 279-286, 2011.

[10] X. Wang and D. Han, "Some identities related to Dedekind sums and the Chebyshev polynomials," International Journal of Applied Mathematics and Statistics, vol. 51, no. 21, pp. 334-339, 2013.

[11] E. H. Doha, A. H. Bhrawy, and S. S. Ezz-Eldien, "Numerical approximations for fractional diffusion equations via a Chebyshev spectral-tau method," Central European Journal of Physics, vol. 11, no. 10, pp. 1494-1503, 2013.

[12] A. H. Bhrawy and M. A. Zaky, "A method based on the Jacobi tau approximation for solving multi-term time-space fractional partial differential equations," Journal of Computational Physics, vol. 281, pp. 876-895, 2015.

[13] E. H. Doha, A. H. Bhrawy, R. M. Hafez, and M. A. Abdelkawy, "A Chebyshev-Gauss-Radau scheme for nonlinear hyperbolic system of first order," Applied Mathematics \& Information Sciences, vol. 8, no. 2, pp. 535-544, 2014.

[14] E. H. Doha and A. H. Bhrawy, "A Jacobi spectral Galerkin method for the integrated forms of fourth-order elliptic differential equations," Numerical Methods for Partial Differential Equations, vol. 25, no. 3, pp. 712-739, 2009.

[15] N. Bircan and C. Pommerenke, "On Chebyshev polynomials and GL(2,Z/pZ)," Bulletin Mathematique de la Societe des Sciences Mathematiques de Roumanie, vol. 103, no. 4, pp. 353364, 2012.

[16] C. Pan and C. Pan, Basic Analytic Number Theory, Harbin Institute of Technology Press, Harbin, China, 2016.

[17] T. M. Apostol, Introduction to Analytic Number Theory, Springer, New York, NY, USA, 1976. 


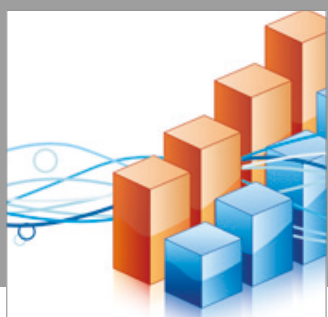

Advances in

Operations Research

vatersals

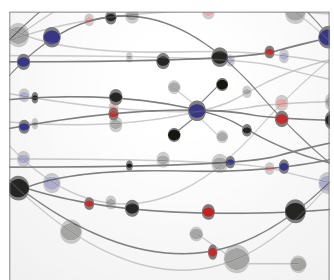

\section{The Scientific} World Journal
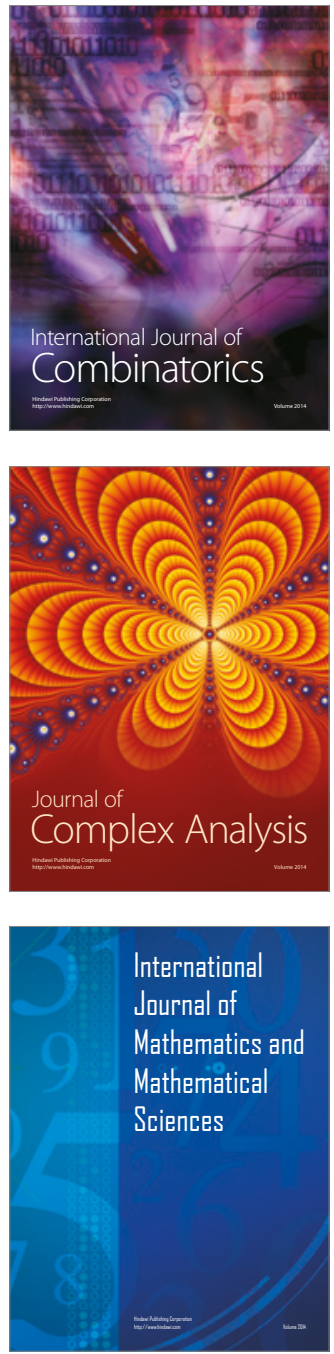
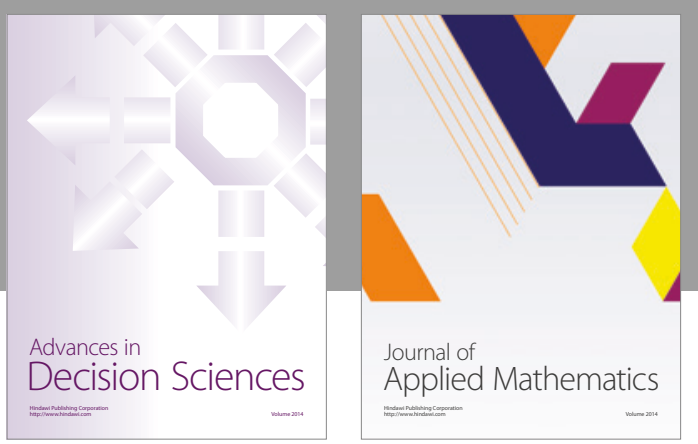

Algebra

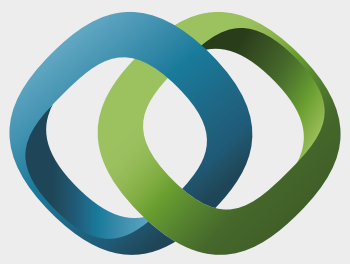

\section{Hindawi}

Submit your manuscripts at

https://www.hindawi.com
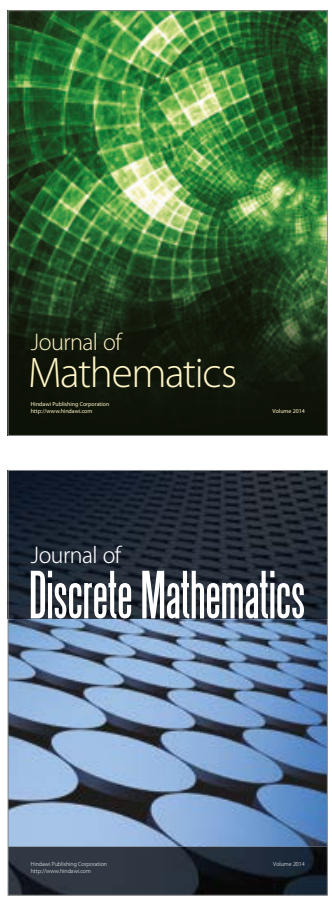

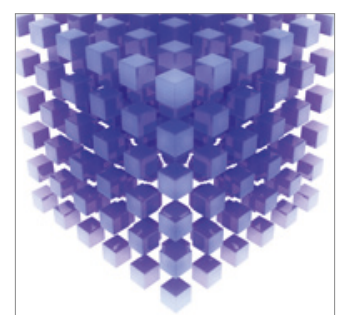

Mathematical Problems in Engineering
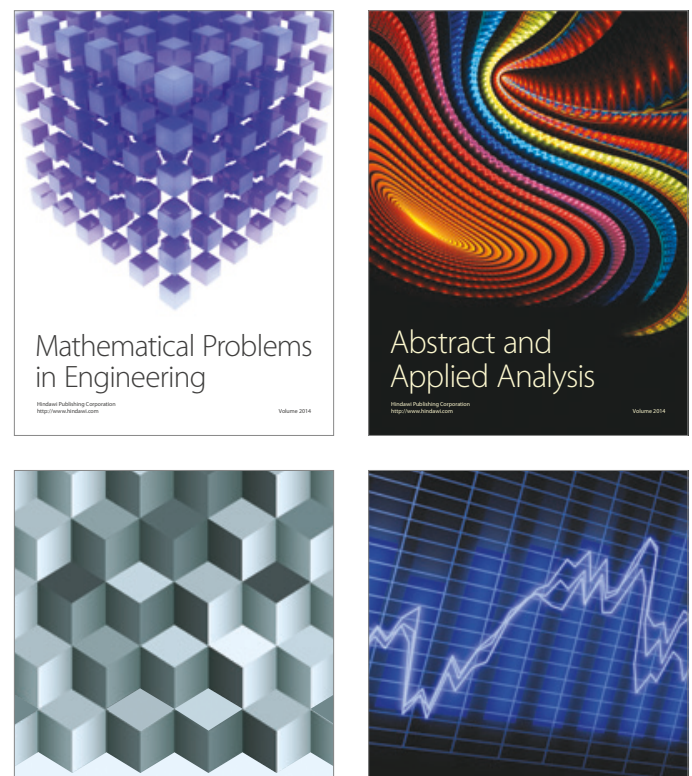

Journal of

Function Spaces

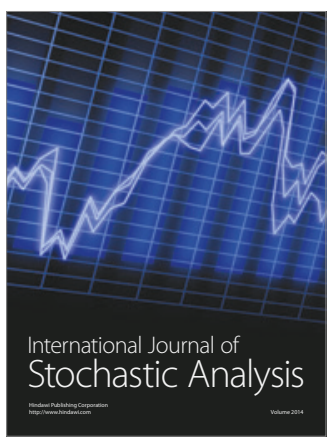

Probability and Statistics
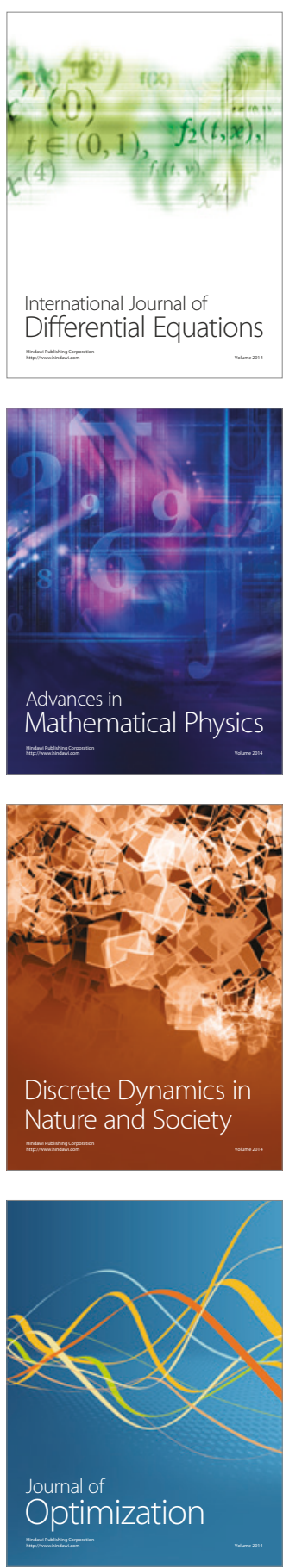